\title{
Balancing Multifibre and Wavelength Converter Cost in Wavelength Routing Networks
}

\author{
P. Pavon-Marino(1), R. Aparicio-Pardo(2), B. Garcia-Manrubia(2), J. Fernandez-Palacios(3), O. Gonzalez(3), F. \\ Martin(3), J. Garcia-Haro(2) \\ 1: Universidad Politécnica de Cartagena, Plaza del Hospital 1, 30202, Cartagena (Spain), pablo.pavon@upct.es \\ 2: Universidad Politécnica de Cartagena, \{rap@alu.upct.es, mbgm@alu.upct.es, joang.haro@upct.es\} \\ 3 Telefonica I+D, Emilio Vargas 6, 28043 Madrid, Spain, \{jpfpg,ogondio\}@tid.es
}

\begin{abstract}
This paper evaluates the balance between the cost of multifibres and the cost of wavelength converters in multilayer networks. A novel ILP formulation of the static planning problem is applied.
\end{abstract}

\section{Introduction}

Multilayer wavelength-routed networks route the higher layer traffic (i.e. IP traffic) onto transparent lightpaths. A node can change the wavelength of a traversing lightpath using one wavelength converter (WC). WCs can avoid wavelength clashing, unblock lightpath requests, and thus increase network capacity. Other option is the multifibre approach. This means that two neighbour nodes in the network, are connected with a bundle of $K$ fibres, $K>1$. Then, at most $K$ lightpaths that traverse the bundle from node $i$ to node $j$, can be assigned the same wavelength, and occupy different fibres in the bundle.

On one hand, the multifibre approach adds the cost of amplifying, equalizing, monitoring, switching, etc. more fibres. On the other hand, the WC approach involves the cost of one WC device per wavelength conversion (we assume that the WCs are shared per node). This paper compares the interest of both approaches in the static planning case. For this, a binary ILP (Integer Linear Programming) formulation is proposed which simultaneously includes the cost of both alternatives. Then, we obtain the minimum cost solution under different conditions, and evaluate the actual use of WCs and/or multifibres. Next section describes the proposed ILP formulation. Section 3 presents the results obtained. Finally, section 4 concludes.

\section{Binary ILP formulation}

Let $N$ be the set of nodes, $M$ the set of unidirectional links. We denote as $|\cdot|$ the number of elements of a set. Each link $m \in M$ is a pair $(i, j), i \in N, j \in N$, composed by a bundle of $K$ fibres. Then, the total number of fibres in the network is given by $|F|=K|M|$. We denote $F_{\text {in }}(n)$ and $F_{\text {out }}(n)$ as the set of fibres that end and start in node $n$, respectively. The same spectral grid of $W$ wavelengths is considered in all the fibres. The cost of activating a fibre $f$ is $c_{f}, f \in F$. The cost of a wavelength converter is given by $c_{W C}$. Let $S$ be the demand of lightpaths to be established. The decision variables to the problem are:
○ $p(s, f, w)=\{1$ if lightpath $s$ traverses fibre $f$ using wavelength $w, 0$ otherwise $\}, s \in S, f \in F, w \in W$.

- $e_{f}=\{1$ if fibre $f$ is active, 0 otherwise $\}, f=1 \in F$

- $c^{+}(s, n, w) \in\{0,1\}, c^{-}(s, n, w) \in\{0,1\}$. These variables are used to track wavelength conversion in node $n$, for lighptath $s$, involving wavelength $w . s \in S$, $n \in N, w \in W$.

The ILP cost function (minimize) and constraints are:

$$
\begin{aligned}
& \sum_{f \in F} c_{f} e_{f}+\frac{c_{W C}}{2} \sum_{s \in S, n \in N, w \in W} c^{+}(s, n, w)+c^{-}(s, n, w), \text { s.t. } \\
& \begin{array}{l}
\text { 1) } \sum_{s \in S} p(s, f, w) \leq 1, \forall f \in F, w \in W \\
\text { 2) } \sum_{\substack{f \in \text { Fout }(n) \\
w \in W}} p(s, f, w)-\sum_{\substack{f \in \text { Fin }(n) \\
w \in W}} p(s, f, w)= \\
\qquad=\left\{\begin{array}{l}
1, \text { if } s \text { starts in node } n \\
-1 \text { if } s \text { ends in node } n, \forall s \in S, \forall n \in N \\
0 \text { otherwise }
\end{array}\right. \\
\text { 3) } p(s, f, w) \leq e_{f}, \forall s \in S, f \in F, w \in W \\
\text { 4) } \sum_{f \in F o u t(n)} p(s, f, w)-\sum_{f \in \text { Fin }(n)} p(s, f, w)=c^{+}(s, n, w)-c^{-}(s, n, w), \\
\forall w \in W, s \in S, s \text { neither starting nor ending in node } n
\end{array}
\end{aligned}
$$

First set of constraints avoid wavelength clashing. Second set are the conservation constraints, allowing wavelength conversion. Third set of constraints avoid any lightpath to traverse a fibre, unless it is active. Fourth set of constraints tackle the wavelength conversion issue. When a lightpath $s$ converts its wavelength in node $n$ from $w_{1}$ to $w_{2}, c^{-}\left(s, n, w_{1}\right)=1$ and $c^{+}\left(s, n, w_{2}\right)=1$. Both are 0 otherwise. As this formulation counts twice each conversion, $c_{W C}$ value is divided by 2 in the cost function.

\section{Results}

We tested three networks of 7 nodes: a mesh network (Figure 1.a), a ring and a star with centre in node 3 (last two cases with fibres of $100 \mathrm{~km}$ length). In all the cases, links have two fibres, and 8 wavelengths per 
fibre. One fibre in the link is already pre-activated at cost 0 . The cost of activation of the other fibre, normalized to one transmitter plus one receiver cost, is taken from [1]: $20+0.96036(d / 80)+1.585(d / 360)$, where $d$ is the link distance and $\mathrm{km}$. Divisions are rounded to the floor, and correspond to optical line amplifiers, and dynamic gain equalizers per each 80 $\mathrm{km}$ and $360 \mathrm{~km}$ spans respectively.

The formulation has been implemented in the MatPlanWDM tool [2], which interacts with a TOMLAB/CPLEX solver [3]. Let $T$ be the $7 \times 7$ traffic matrix in Figure 1, obtained from traffic forecast studies for a national optical backbone (measured in Gbps). All the traffic matrixes used are calculated multiplying $T$, by a real factor. For each topology we made 20 tests with different traffic matrixes, from the higher traffic carried at 0 cost, to the higher carried traffic feasible found. In each test, we apply the heuristic algorithm in [4] which obtains the minimum set of lightpaths which is able to carry the traffic demand, assuming a capacity of 10 Gbps per lightpath. As the WC technology is not fully mature, WC prices were estimated sweeping the WC cost from 0.01 ( $1 \%$ of a transmitter plus a receiver), to a sufficiently high value. Naturally, we expected a lower preference for WCs, as the WC cost grew. Surprisingly, as it is shown in Figure 1, WCs were not used in any case, even considering the lower WC cost of $c_{w c}=0.01$. As traffic grew, a higher number of fibres were activated. But always happened, that given a set of active fibres, if a solution with WCs was found, a solution without WCs and the same active fibres was found (obviously at a lower cost).

Previous studies showed that multifibre networks could reduce at a great extent the need of WCs [5] for dynamic planning scenarios. Our tests correspond to static planning. It seems that the advantage of a deterministic knowledge of the traffic (in contrast to dynamic planning), greatly favours finding minimum solutions without WCs. After these results, we conducted more tests in these topologies which confirmed that minimum cost solutions with WCs in static planning are largely infrequent, and appear in very narrow intervals of traffic demands.

\section{Conclusions}

In the realistic scenarios tested, for static planning, results neglect the interest of WC devices to increase network capacity. Multifibre option is utterly preferred.

\section{Acknowledgements}

The work described in this paper was carried out with the support of the BONE-project ("Building the Future Optical Network in Europe"), a Network of Excellence funded by the European Commission through the 7th ICT-Framework Programme, and the support of MEC Spanish project TEC2007-67966-01/TCM CONPARTE-1.

\section{References}

1. NOBEL phase 2 -Deliverable D 2.4. "Migration Guidelines with Economic Assessment and New Business Opportunities Generated by NOBEL phase 2": http://www.istnobel.org/Nobel2/imatges/D2.4_final\%20version.p df.

2. P. Pavon-Mariño et al., MatPlanWDM: An educational tool for network planning in wavelength-routing networks, Lecture Notes in Computer Science, vol. 4534 (2007), pp. 58-67.

3. http://tomopt.com/tomlab/ (last access April 2008).

4. V.R. Konda et al., Algorithm for Traffic Grooming in Optical Networks to Minimize the Number of Transceivers," IEEE Workshop on High Performance Switching and Routing, (2001), pp. 218-221.

5. L. Li et al., A new analytical model for multifiber WDM networks, IEEE J. Select. Areas Commun., vol. 18 (2000), pp. 2138-2145.

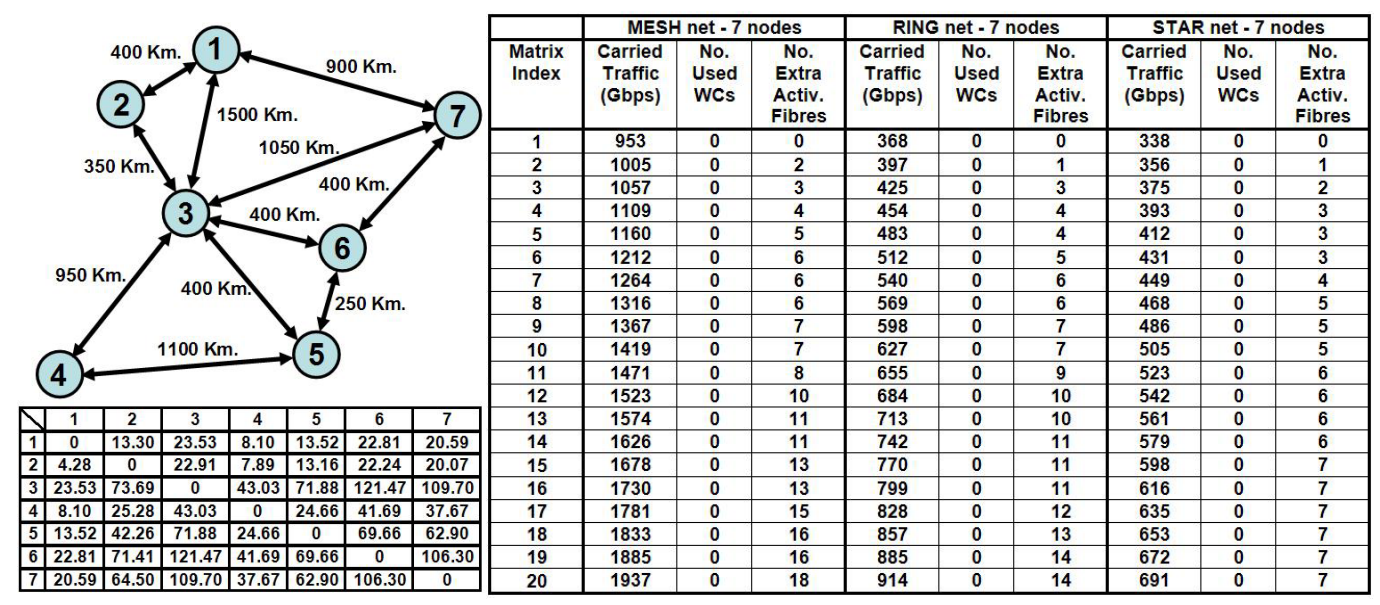

Figure 1. Mesh topology and traffic matrix T (left side). Table of results (right side). 